ÉGYPTE monde arabe

\section{Égypte/Monde arabe}

$3 \mid 2000$

La censure ou comment la contourner

\title{
Censure et sens commun en Égypte
}

Ou l'invention répétée de la normalité islamique

Jean-Noël Ferrié

\section{(2) OpenEdition}

Journals

Édition électronique

URL : https://journals.openedition.org/ema/787

DOI : $10.4000 /$ ema.787

ISSN : 2090-7273

Éditeur

CEDEJ - Centre d'études et de documentation économiques juridiques et sociales

Édition imprimée

Date de publication : 30 juin 2000

Pagination : 49-61

ISBN : 2-87027-856-X

ISSN : 1110-5097

Référence électronique

Jean-Noël Ferrié, «Censure et sens commun en Égypte », Égypte/Monde arabe [En ligne], 3 | 2000, mis en ligne le 08 juillet 2008, consulté le 07 juillet 2022. URL : http://journals.openedition.org/ema/787 ; DOI : https://doi.org/10.4000/ema.787

Ce document a été généré automatiquement le 7 juillet 2022.

Tous droits réservés 


\title{
Censure et sens commun en Égypte
}

\author{
Ou l'invention répétée de la normalité islamique
}

Jean-Noël Ferrié

1 La censure est une pratique égyptienne suffisamment notable pour être étudiée en soi. Toutefois, cette étude présente de nombreux écueils dont le moindre n'est pas la dénonciation libérale des activités liberticides des hommes de religion (rigâl al-dîn). Je ne doute pas qu'ils fassent tous les efforts nécessaires en ce sens; je doute, en revanche, que l'étude de la censure puisse se satisfaire du repérage des groupes sociaux et des institutions engagées dans des « entreprises morales » (Becker, 1985). En effet, ceci ne nous permet guère de comprendre ce qui fait que la censure paraisse normale aux acteurs, puisqu'il est très rare - en Égypte comme ailleurs - qu'elle soit radicalement mise en cause, y compris chez les « libéraux ». Bien souvent les débats portent sur les objets, les cas d'espèce et, rarement, sur la justification de la censure en générale (sauf en ce qui concerne, peut-être, la censure politique). C'est que celle-ci paraît moins l'émanation d'une volonté autoritaire que l'expression du « sens commun ». De ce point de vue, il peut paraitre intéressant, dans le cas égyptien, d'interroger la validation religieuse de la censure des mœurs et des idées sur les mœurs. Représente-t-elle vraiment l'application d'interdits divins ou ressortit-elle d'un autre système d'ordre? De manière plus provocante, la question peut aussi être posée de la manière suivante : si l'on parvenait à priver les hommes de religion de leur influence modifierait-on du même coup l'attitude des gens vis-à-vis de ce qu'il est convenable de publier?

2 C'est dans cette perspective que je m'intéresse à la place de l'islam dans l'organisation de la censure. Je vais en discuter, ici, en proposant un cadre conceptuel permettant d'en rendre compte. Ce cadre conceptuel ménage une place particulière au «sens commun » conçu, non comme un contenu - ce qui pourrait être, par exemple, la culture commune des Égyptiens ou le sens commun comme culture, pour reprendre une expression de Geertz -, mais comme une procédure. Le sens commun, c'est ce qui paraît normal aux gens, quel que soit l'objet en question. C'est, si l'on préfère, l'acquisition d'un statut de vérité et non la sélection d'un ensemble de vérité. J'en traite longuement tout au long de l'article, tentant de montrer comment l'islam n'a pas exactement, dans cette matière, le rôle directeur que l'on se plaît à lui donner. 
Le point de vue du cheikh

3 L'ancien cheikh d'al-Azhar déclarait ainsi, à propos de la discussion et de sa censure dans les médias modernes :

«La philosophie a ses lois et celui qui s'y adonne doit être un spécialiste comme dans les autres sciences; c'est pourquoi elle ne doit pas être abandonnée à la multitude. Diffusée dans le public par des pseudo-philosophes, elle n'est que tromperie et n'a rien à voir avec la religion. De même, ceux qui tirent argument de l'existence, dans le passé, de sociétés de philosophes, oublient avec quelle facilité les médias dont nous disposons aujourd'hui répandent pour le meilleur et pour le pire des informations et des idées parmi lesquelles il est difficile de séparer le bon grain de l'ivraie. Il n'y a donc aucune raison de protester d'un passé où la discussion philosophique avait lieu entre initiés et ne touchait pas les masses. »

De ce point de vue, la publicité de la référence à l'islam ne doit pas déboucher sur une discussion publique car le lieu de la référence n'est tout simplement pas le lieu du débat. Cela dit explicitement que la référence ne se fonde aucunement sur un savoir situé et discutable. Sur quoi s'établit-elle alors? Là aussi, les propos de l'ancien cheikh sont clarifiants :

« La créativité ne permet pas à un écrivain de dévier du bon sens, lorsqu'il parle ou écrit à propos de ce qui constitue le cœur de la croyance, d'autant que s'il fait cela, il porte également atteinte aux sentiments des citoyens et à leur foi et se moque de leur raison et de leur intelligence, alors qu'il devrait respecter et protéger les valeurs comme cela est admis dans toutes les croyances. "

«La censure n'est pas une fin en soi, mais le moyen de vérifier que les écrits respectent ce qui est socialement sain, la croyance, les mœurs, les valeurs attachées au comportement. Elle vise à écarter [de la société] tout ce qui nuit, de sorte que la jeunesse ne dérive pas vers des idées extérieures aux principes de l'islam et de sa sharia. $"^{5}$

La référence s'établit sur les croyances du sens commun, sur les sentiments normaux des personnes et non pas sur leurs compétences philosophiques. Elle assimile en outre la définition (censément) commune de la vie saine et des bonnes mœurs à la religion elle-même:. Ce qui se passe ici demande à être retenu dans sa particularité : l'indexation islamique (le résultat de la référence) des évaluations et des catégories relevant du sens commun opère le rabattement de la normalité religieuse sur le sens commun lui-même, permettant d'assimiler au prescrit religieux des jeux de conceptions communes qui n'en relèvent pas de manière déterminée. Cette adéquation entre normalité (ici religieuse) et sens commun constitue ce que Baudouin Dupret et moi-même nommons la «communalité ». La référence est l'opérateur de la «communalité », puisqu'elle indexe une opinion commune ou un comportement commun à une norme, donnant comme réalité de la norme l'effectivité des pratiques qu'elle choisit d'indexer. Prenons un exemple d'enchaînement en "communalité » : «selon le Coran, la femme ne doit pas travailler; le travail féminin est, d'ailleurs, l'une des causes du chômage des hommes ». La référence islamique permet ainsi d'assimiler à des dispositions coraniques sur les femmes une conception toute moderne - puisque née de la situation présente - de leur place. Au demeurant, l'usage de la référence ne relève pas forcément d'un dessein conservateur. Ainsi, le détour par la «communalité " peut servir, aux magistrats de la Haute Cour constitutionnelle égyptienne, à justifier coraniquement l'interdiction du port du voile complet (niqâb) en tant qu'il est contraire aux us égyptiens contemporains'. Il s'agit d'une position incontestablement réformistes, qui procède pourtant de la même pratique indexicale que celle qui consiste à référer à 
l'islam l'explication du chômage des hommes par le travail des femmes. L'une et l'autre position sont ainsi fondées sur le sens commun.

Qu'est-ce que le sens commun?

6 L'utilisation du «sens commun » par les acteurs est prédicative puisqu'elle affirme quelque chose à propos de ce que le «sens commun" croit. Elle affirme substantiellement cette chose même si son contenu est vague. En disant que «les Égyptiens aiment les bonnes mœurs ", un locuteur (le cheikh d'al-Ahzar, par exemple) affirme une qualité substantielle des Égyptiens, même si le contenu exact du terme «bonnes mœurs » demeure jusqu'à un certain point énigmatique. Jusqu'à un certain point seulement et seulement si l'on entend obtenir une définition précise des « bonnes mœurs » et de ce qui fonde la décision qu'elles sont cela plutôt qu'autre chose. En effet, n'importe quel membre normalement socialisé de la société égyptienne - fut-il libertin ou amoral - comprendra que l'expression sert à désigner, en général, les attributs d'une sexualité conventionnelle et les caractéristiques d'une présentation de soi convenable.

7 L'utilisation du « sens commun » par l'analyste porte précisément sur ce que les acteurs reconnaissent typiquement comme une chose admise quel que soit son contenu, en d'autres termes sur des énonciations qui ne troublent pas le cours d'un échange parce qu'il serait difficile pour l'un des interlocuteurs de comprendre exactement à quelle sorte de chose il est fait référence. Si je dis, par exemple : «mari et ... », puis que je laisse ma phrase en suspens, la personne à qui je parle pourra ajouter, à part elle : « et femme » sans prendre beaucoup de risques. Elle ne dira pas : «mari et cheval» ou «mari et trombone». Ce qui relève du sens commun, du point de vue de l'analyste, c'est donc la reconnaissance de cette standardité (et non pas son contenu).

Le quatuor de la censure

8 Revenons maintenant à la déclaration du cheikh d'al-Azhar : elle affirme en substance que les créateurs doivent respecter les croyances de leurs concitoyens et ne rien faire qui puisse égarer la jeunesse. Un autre intervenant dans le débat sur la censure dit (toujours en substance) que la liberté du créateur n'est pas absolue : elle est limitée par sa capacité à présenter des idées claires et perspicaces". Un troisième soutient que le droit d'expression doit être limité parce que l'audience des créateurs est considérable et qu'ils doivent alors prendre en compte la décence, la courtoisie et le goût". Un quatrième déclare que la liberté ne peut être totale parce que cela serait socialement irresponsable dans une société où l'opinion publique n'est pas encore mûre ${ }^{n}$. Les quatres positions sont défendues par des personnalités contrastées dont trois - c'est-àdire toutes à l'exception du cheikh d'al-Azhar - représentent un courant " progressiste». Cependant, ces quatre personnalités convergent bien plus qu'elles ne divergent. Mais, en fait, de quelle manière et sur quoi pouvons-nous nous fonder pour l'affirmer ? À ce point, il ne s'agit donc pas de retourner à une erreur de qualification et de considérer ce qu'écrivent les acteurs comme un ensemble d'éléments pertinents pour décrire les procédures qu'ils mettent en œuvre. Ou, si l'on préfère, les références qu'ils font - à l'islam ou au bon sens - ne sont pas à l'origine des catégorisations auxquelles ils procédent. Ainsi, la question qui convient n'est-elle pas : « qu'est-ce qu'ils pensent de la censure? » ou «pourquoi donc écrivent-ils ce qu'ils écrivent?» mais seulement: «dans quel dispositif de catégorisation se situent-ils au moment où ils écrivent?»

9 Les personnalités intervenant dans le débat sur la censure procèdent toutes à une catégorisation des créateurs comme personnes « responsables ». En d'autres termes, le 
dispositif de catégorisation qu'ils utilisent porte sur la moralité de la personne, c'est-àdire sur sa capacité à reconnaître spontanément ses obligations. Certes, elles peuvent être conçues différemment mais ceci - qui est le contenu à proprement parler - ne nous intéresse pas. Venons-en donc au dispositif de catégorisation des créations. Il se fonde sur la distinction de ce que l'on peut publier et de ce qui ne peut l'être. Il comprend la collection des choses publiables, avec comme catégories le «sain », le «moral» ou le " convenable » et la collection des choses non publiables". Jusqu'à un certain point, ces collections se recoupent en incluant symétriquement des catégories opposées. Si nous revenons maintenant aux dispositifs de catégorisation des quatre intervenants du débat sur la censure, nous remarquons qu'ils convergent : les créateurs sont catégorisés comme des êtres moraux ou non moraux et les créations comme des choses publiables ou non publiables. Parmi ces protagonistes, seul le cheikh d'al-Azhar prétend référer son dispositif à la religion. Cela ajoute-t-il quelque chose à la pertinence (sociale) de son discours? Les trois autres protagonistes considèrent, eux, que leur dispositif de catégorisation est suffisamment clair et partagé pour être, tel quel, compris de tous et admis.

10 Pourquoi, cependant, pensent-ils être à même de se passer de la référence islamique ? C'est sans doute la réponse la plus aisée à apporter. En effet, nous pouvons partir du constat que les collections de catégories - la liste des typifications des choses et celle des attributs moraux typiquement nécessaires au travail de créateur - ont un « air de famille ", indépendamment du fait qu'elles soient ou non référées à l'islam. Le détour par le dispositif de catégorisation met ainsi en lumière le fait que les opérations de catégorisations auxquelles se livrent les acteurs s'ancrent dans un monde commun sans être, pour autant, communément religieuses. Cette façon d'attribuer des droits et des devoirs comme de distinguer le publiable de l'impubliable et d'en établir la distinction par des collections de catégories fait partie des choses que les acteurs reconnaissent avec netteté. De ce point de vue, la référence islamique n'est pas nécessaire - et c'est désormais une évidence - pour établir la « communalité ».

À quoi sert la référence?

11 Il reste cependant à répondre à la question : « qu'ajoute cette référence ? » Nous étions fondés à croire, dans un premier temps, qu'elle établissait la «communalité » des conduites qu'elle indexait. Mais il est vite apparu que la « communalité » pouvait être établie de façon endogène, en décrivant la régularité des procédures impliquant des dispositifs de catégorisations comme preuve de l'existence d'un ordre des choses ou d'une nature des choses, c'est-à-dire en fondant le sens commun comme référence sur le sens commun comme procédure. Ce faisant, nous ne nous situons plus dans la description de l'analyste mais dans une rhétorique d'acteur. Cette rhétorique consiste à tirer argument de la stabilité apparente des procédures gouvernant les échanges sociaux pour affirmer qu'elle exprime la nature des choses. On retrouve ici la position de Durkheim : «C'est, en effet, un postulat essentiel de la sociologie qu'une institution humaine ne saurait reposer sur l'erreur et sur le mensonge : sans quoi elle n'aurait pu durer. Si elle n'était fondée dans la nature des choses, elle aurait rencontré dans les choses des résistances dont elle n'aurait pu triompher. » $(1985$, p. 3)

Toutefois, la nature des choses ne suffit pas à justifier de leur état : elle ne les place pas d'emblée dans la perspective de ce qui vaut, elle ne permet pas d'en discuter comme de ce qui est bon pour soi et pour les autres, comme d'un accomplissement. Elle institue, en quelque sorte, une normalité sans téléologie. De ce point de vue, la référence 
islamique pourrait relever de ce que Jon Elster nomme "l'obsession du sens ", c'est-àdire, de « la tendance répandue à chercher le sens de tous les phénomènes » (1986, p. 90 sqq.). L'imputation de sens est sans doute une condition nécessaire pour faire de l'ordre des choses un argument de débats. En effet, ainsi que le remarque Jean-Marc Ferry (1992, p. 122) : « Nous avons ce besoin "idéaliste", fondé dans la situation de parole ellemême, de pouvoir honorer l'exigence d'une validité qui, en tant que prétention, transcende les contextes. " "Or, s'il est possible de soutenir jusqu'à un certain point que la nature des choses organise les contextes, il est difficile d'affirmer en même temps qu'elle les transcende, dans la mesure où elle leur est coextensive. Elle représente la raison à même les contextes, la confusion de la norme et du fait. Or, c'est le ridicule propre de Pangloss de n'être philosophe que pour assurer que «tout est pour le mieux» du seul fait d'être. Cette non-discrimination totale ne correspond pas à la pratique des acteurs et nous avons vu que la "communalité » était affirmée sélectivement. Le débat sur la censure ainsi que le jugement sur le port du voile complet exprimaient ce principe de discrimination: il y a des choses normales et d'autres qui ne le sont pas ou, en d'autres termes, tout n'est pas normal en même temps. La référence islamique permet de fonder une discrimination entre les objets du sens commun. Elle la fonde dans le sens précis où elle la justifie et non où elle l'initie.

L'incertitude du censeur bien intentionné

13 La normalité islamique, en effet, n'est pas déduite de la référence coranique mais de la " communalité ", c'est-à-dire de la normalité du commun ; elle est ensuite - et ensuite seulement - référée à la normalité islamique, parce qu'elle opère une sélection dans le monde commun et que cette sélection, arbitraire du point de vue de la communalité, ne peut qu'être justifiée extérieurement à ce monde. C'est uniquement à ce moment-là que la référence islamique doit être considérée comme un discriminant, c'est-à-dire comme un ressort d'ordre.

Prenons, par exemple, l'idée selon laquelle on ne peut montrer une "scène d'amour ", dans un film égyptien, et même pas un baiser (plus exactement un french kiss). Une actrice peut être, par exemple, amenée à un commissariat de police et risquer (ou sembler risquer) l'emprisonnement, si un censeur décide, lors de la projection d'un film, que l'acte à laquelle elle se livre (le french kiss) est contraire aux bonnes mœurs. Cette idée que les bonnes mœurs impliquent un comportement pudique et interdisent le baiser relève pour le censeur de la normalité du commun plutôt que simplement de l'islam. Comment justifier ce point? Par l'argument des versions et des variations (Ferrié, 1998). Selon cet argument, les modèles de pratiques existent en plusieurs versions clairement discriminées selon les contextes. Une "scène d'amour " ne peut être filmée, en Égypte, dans sa version qu'il est tentant de qualifier de normale, c'est-àdire dans la version qui permette de décider que les acteurs sont en train de représenter une relation sexuelle plutôt qu'une étreinte. Jusqu'à un certain point, l'étreinte est une version autorisée de la scène d'amour mais elle doit néanmoins se soumettre à certaines contraintes et, le plus souvent, à des contraintes précises. Ces contraintes intervenant à l'intérieur de la version, déterminent ses variations. Combien de temps doit durer l'étreinte? Que ne peut-on faire durant celle-ci ? Doit-on montrer les lèvres en train de se joindre ou le plan doit-il simplement montrer le contact des visages? Les variations posent, on le comprend aisément, un véritable problème, cognitif certainement plus qu'éthique, au censeur car elles démultiplient les cas 
d'espèces. Dès lors que l'on est engagé dans le contrôle des variations, cela signifie que la version est admise et qu'une gamme considérable de variations le sont aussi.

Est-ce alors l'argument coranique qui permet à lui seul de définir quelle variation est admissible et quelle variation ne l'est pas? On peut légitimement douter que le prescrit divin permette cette précision. De ce qui concerne, plus ou moins clairement, la pudeur de la femme dans le Coran à la détermination de la durée d'un baiser ou de la pose des acteurs au moment du baiser et à la vigueur de leur enlacement, il y un espace qu'aucun effort herméneutique ne peut espérer franchir. L'interprétation a ses limites (Eco, 1990). Sur quoi se fonde alors le censeur pour censurer ? Sur sa détermination de la « communalité » de la durée et des poses admises pour un baiser, c'est-à-dire sur rien d'autre qu'une procédure de catégorisation qu'il objective en arguant de sa normalité. Cette détermination est à la fois commune parce qu'elle implique des compétences partagées (on retrouve, ici, le sens commun comme procédure) et, quoiqu'il puisse prétendre, individuelle parce qu'elle implique un travail d'explicitation de la part de l'acteur (on retrouve, alors, le sens commun comme référence). Cette opération faite, le censeur pourra alors justifier son choix - le choix d'une durée moyenne et d'une pose en le référant directement au prescrit coranique, c'est-à-dire en mettant entre parenthèses les opérations contingentes qui l'ont conduit à censurer un baiser plutôt qu'un autre. Une même observation peut être faite en ce qui concerne la censure des magazines de modes étrangers et principalement les magazines anglais qui contiennent souvent des photos sophistiquées et provocantes. Comment le censeur fait-il alors, s'attaquant à un magazine"s, pour décider que des seins nus sans visage sont tolérables tandis que d'autres, accompagnés d'un visage, ne le sont pas, pourquoi arrache-t-il une page et pas l'autre?

L'islam comme construction du sens commun

16 Du point de vue du débat, la censure de la nudité est très généralement présentée comme ressortissant du respect de l'islam. Lors d'une séance de l'Assemblée du Peuple, le député indépendant Galâl Gharîb reprocha au ministre de la Culture d'avoir laissé éditer, par un organisme placé sous sa tutelle, une revue où figurait la reproduction d'une œuvre de Klimt représentant Adam et Eve nus. À ce moment, un autre député s'écria : "Au pays d'al-Azhar?» L'anecdote ${ }^{16}$ met bien en évidence la référence islamique fondant la nécessaire censure de la nudité. Il n'est pas question de nier la présence de cette référence mais, seulement, de bien préciser son ordre d'intervention et la part qui lui revient dans la détermination des actions des personnes qui s'y réfèrent. Il est certes possible de soutenir que les personnes s'y réfèrent dans la démarche même qui les pousse à censurer la nudité. Dans ce cas, cependant, l'objection de la détermination des versions et des variations me paraît réellement difficile à lever : la "communalité » est, de toute évidence, première. En revanche, on peut soutenir que cette "communalité » est, elle-même, construite avec de l'islam et qu'on se réfère alors à l'islam en se fondant sur l'évidence de l'islamité de ce que l'on réfère. Certainement est-ce vrai du point de vue de la façon dont un acteur commenterait les raisons de sa référence, s'il avait à le faire. Mais cela ne change rien au fait que ce qu'il nomme "islam», dans ces circonstances, n'est rien d'autre qu'une position de sens commun et non un savoir d'expert, déduit d'une confrontation directe aux textes.

La référence à l'islam comme norme se fonde ainsi - comme l'a montré Baudouin Dupret, à propos de la référence plus spécifique à la Loi divine" - sur une connaissance de sens commun de l'islam, en tant que telle indistincte des autres éléments du sens 
commun parce que toujours rapportée aux circonstances de la vie quotidienne dans lesquelles l'islam est mentionné. Cette façon de faire possède une propriété que l'on pourrait qualifier d'» agglutinante » : l'islam se construit, en somme, par agglutination des déterminations de sens commun qui lui sont référées. Toutefois, alors que l'agglutination est une procédure de sens commun - en d'autres termes : une procédure implicite - la critique de l'agglutination implique, elle, de recourir à une procédure démonstrative fondée sur l'exégèse de la référence elle-même. Il s'agit, en effet, de montrer que telle ou telle chose communément décrite comme étant de l'islam (ou décrite comme en étant communément) n'est pas et ne sera jamais de l'islam, c'est-àdire qu'il s'agit d'agir contre l'évidence, l'allant-de-soi, et donc de substituer le débat à l'accord tacite, autrement dit il est question d'entrer dans une épreuve (Boltanski \& Thévenot, 1991, p. 68 sqq.).

Le sens commun fait la Loi

Indéniablement, nous avons alors affaire à une procédure asymétrique qui établit, cependant, une contrainte argumentative pour les acteurs. Dans le dispositif décrit, c'est seulement l'engagement $d u$ débat qui implique l'explicitation du contenu islamique de la référence et donc, l'obligation pour celui qui s'y engage de faire ses preuves à l'intérieur de ce registre argumentatif, parce que les positions auxquelles il s'attaque sont seulement adossées à cette référence. Tout au moins le croit-il mais il s'agit d'une erreur (au sens d'une mauvaise stratégie).

Disons simplement, ici, que la critique de la référence islamique devra déployer une argumentation coranique suivie et généralement d'autant plus fouillée qu'elle s'oppose à une position convenue. Mais, en admettant même que la pertinence de la référence soit ponctuellement invalidée - ce qui n'est jamais certain -, il restera toujours à invalider la « communalité » sur laquelle elle s'adosse et qui, construite par autre chose que la référence, ne peut être mise en jeu par sa simple invalidation. En revanche, chaque attaque contre la pertinence d'une référence permettra aisément à ses partisans d'établir la validité coranique de celle-ci en précisant, par exemple, le verset coranique auquel il convient de se référer, en donnant, en quelque sorte, la règle à suivre. Au lieu d'évoquer «le pays d'al-Azhar " pour condamner la nudité, ils citeront tel passage du Coran, tel commentaire (tafsir) ou tel opinion de jurisconsulte. Mais il est douteux - nous l'avons vu - qu'ils puissent aisément, par déduction suivie, expliquer le cas d'espèce lui-même. Comme il est assez douteux que leur position se fonde sur des compétences philosophiques étendues, ainsi que le soulignait le cheikh d'al-Azhar. (Et même si c'était le cas, l'application au cas d'espèce se heurterait toujours à l'obstacle des versions et variations). En revanche, la référence coranique explicitée pourra servir à former des séries analogiques robustes, à mettre ensemble des éléments sans lien anté-prédicatif mais présentant, du point de vue du contexte, un "air de famille » (c'est le mécanisme de l'agglutination).

L'exemple qui vient à l'esprit est celui de la fornication (zinâ). La fornication consiste dans le fait d'avoir des relations sexuelles avec un partenaire illégitime, c'est-à-dire aujourd'hui un partenaire autre que l'époux ou l'épouse (Bousquet, 1990, p. 69 sqq.). La fornication est un crime sanctionné figurant dans le Coran (hadd, plur. hudûd)". Dans sa forme la plus grave, elle peut être punie de la peine de mort par lapidation. Cependant, l'administration de la preuve est malaisée, comme c'est généralement le cas pour les crimes mentionnés dans le Coran". Il faut quatre témoins, mâles, pubères, libres, sains d'esprits et dignes de confiance ayant vu, en même temps, le sexe du fornicateur 
" comme le style dans le pot de collyre $»^{*}$. L'aveu est valable mais il doit être maintenu par le coupable jusqu'à la fin de l'exécution de la sentence ${ }^{4}$. De ce point de vue, le crime de fornication ne peut quasiment pas être sanctionné. Le magistrat égyptien Sayyid al'Ashmâwî en a tiré argument en faveur de l'inapplicabilité intrinsèque de la Loi divine en matière pénale (al-cAshmâwî, 1988). Il s'agit, certes, d'une position de débat mais, même en admettant que la Loi divine pénale soit applicable, elle ne peut l'être que difficilement et, certainement, dans un nombre réduit de cas. Or, nous disposons néanmoins d'une tentative de réforme $d u$ droit pénal égyptien, prétendant à l'application de la Loi divine, c'est-à-dire incorporant, dans ce cas, les crimes et les peines figurant dans le Coran au droit positif (Jacquemond, 1986). Ce qu'il est intéressant de constater est le nombre et la sévérité des articles se rapportant à la police des mœurs. Ceux-ci ne sont pas à proprement parler inscrits, d'une manière ou d'une autre, dans la Loi divine. En fait, l'incorporation au droit du crime coranique de fornication permet d'indexer par rapport à celui-ci et donc de sanctionner sévèrement tout un ensemble de conduites qui n'en relevaient pas anté-prédicativement.

La censure comme stabilisation du sens commun

21 Il serait possible de donner d'autres exemples et, certainement, de pousser plus loin l'analyse mais peut-être vaut-il mieux résumer à présent le cours d'une argumentation dont on ne peut qu'espérer qu'elle ne fut pas contournée. L'une des particularités notables de la référence islamique semble être son ubiquité. Comment alors la définir? Sans doute, dans un premier temps, par le fait qu'elle s'apparente davantage à une forme qu'à un contenu, à une procédure qu'à une substance. Des slogans comme : «l'islam est la solution» illustrent cette caractéristique. Pourtant, la théorie de la forme indéfiniment substantialisable, malgré son intérêt, ne permet pas de rendre compte du caractère indéniablement contraignant de la référence islamique. Si tous les contenus étaient possibles, l'islam ne serait rien et même pas une référence. Il faut donc que les contenus soient limités. L'idée que cette limitation serait imposée de l'intérieur même de la référence se heurte au fait que la contrepartie de l'ubiquité, c'est l'inscription de la référence dans des débats dont elle ne détermine pas le contenu. En d'autres termes, le discours référé est construit ailleurs. Il ne faut pas entendre ce terme - «ailleurs »- comme indiquant, par exemple, que le discours serait construit à l'intérieur d'une configuration idéologique précise ou même de plusieurs, puis légitimé à l'aide de l'islam. L'ubiquité de la référence implique bien autre chose, et c'est sans doute l'une des erreurs de l'analyse (française et anglo-américaine) en science politique que de s'être focalisée sur l'islam en tant que ressource de légitimation ou de dénonciation. La principale conséquence de cette erreur fut d'adosser (paresseusement) la pertinence sociale de la référence islamique à la "tradition musulmane", plus que rapidement considérée à travers quelques ouvrages d'orientalistes. Il ne pouvait en être autrement: sur quoi fonder la légitimité d'une ressource de légitimation sinon sur un donné extérieur aux circonstances de son utilisation?

La démarche peut sembler juste ; elle ne l'est pas. D'abord, elle ne fait que paraphraser, à partir d'une position ironique, le discours des acteurs eux-mêmes. Ensuite, elle reproduit l'erreur logique qui consiste à tenter de fonder une chose dans l'antériorité (ou simplement d'admettre qu'elle s'y fonde), c'est-à-dire à entrer dans un processus de régression infinie ou à arrêter celui-ci de manière arbitraire ${ }^{n}$. C'est pourquoi presque toutes les considérations sur l'islam contemporain sont introduites par des propos de 
longueurs variées, convenus et fastidieux sur l'origine de l'islam ou sur l'islam classique $^{s}$. Nous avons vu, cependant, que la légitimité fondatrice - si l'on peut continuer à user valablement de ce vocabulaire - était produite par la communalité elle-même. Les dispositions et les arguments communs sont ensuite - et seulement ensuite - référés, par leurs promoteurs, à la normalité islamique, parce que ceux-ci opèrent une sélection dans le monde commun et que cette sélection - la leur -, arbitraire du point de vue de l'égale communalité d'autres choses (d'autres arguments ou d'autres dispositions), ne peut être justifiée qu'extérieurement à ce monde. Certes, ici aussi l'islam permet, d'une certaine manière, de produire de « la légitimité » mais il s'agit d'une légitimité seconde et sans doute secondaire, dans la mesure où la référence à l'islam sert, d'abord, à introduire une contrainte argumentative, fondée sur le principe de pertinence islamique, qui oblige les contradicteurs à exciper d'un savoir supérieur à celui mis en œuvre par les partisans du point de vue qu'ils contredisent. Elle sert, ensuite, ainsi que nous l'avons vu, à former des séries analogiques robustes, mettant ensemble des éléments sans lien anté-prédicatif qui présentent, dans un contexte particulier, un «air de famille » et dont la liaison ne peut aussi être réfutée qu'à l'aide d'une science coranique sûre et admissible.

En ce sens, la référence islamique est beaucoup plus qu'un simple moyen de légitimation et beaucoup moins que l'ombre du Coran dans la vie quotidienne : elle est un dispositif permettant de produire de l'islam à partir du sens commun, c'est-à-dire de soustraire à l'épreuve de la discussion - en les investissant d'une autorité telle que seul un langage qualifié peut entreprendre de les invalider - des matières qui normalement ressortissent de la juridiction commune non moins que de l'appréciation individuelle. Dans ce cadre, la censure n'est rien d'autre qu'une procédure pour stabiliser le sens commun.

\section{BIBLIOGRAPHIE}

Albert H., 1968, Traktat über kritisch Vernunft, Tübingen, Mohr.

Apel K. O., 1981, « La question d'une fondation ultime de la raison », Critique, n 413, octobre.

Al-cAshmâwî S., 1988, La Loi divine islamique et le droit positif égyptien (en arabe), Le Caire, Madbûlî.

Becker H., 1985 (1963), Outsiders, Paris, Métailié.

Bercher L., 1926, Les délits et les peines de droit commun prévus par le Coran, Thèse de droit, Paris.

Boltanski L. \& Thévenot L., 1991, De la justification, Paris, Gallimard.

Botiveau B., 1993, « Penser, dire, interdire. Logiques et enjeux de la censure des écrits en Égypte ", Égypte/Monde arabe, $\mathrm{n}^{\circ} 14$.

Bousquet G.-H., 1990 (1966), L'Ethique sexuelle de l'islam, Paris, Desclée de Brouwer.

Chaumont E., 1996, «Compte-rendu de Bernard Botiveau : Loi islamique et droit dans les sociétés arabes », Bulletin critique des Annales islamologiques, vol. 12. 
Dupret B. :

- 1995a, « La sharî a comme référent législatif. Du droit positif à l'anthropologie du droit », Revue interdisciplinaire d'études juridiques, $\mathrm{n}^{\circ} 34$.

- 1995b, « Entre le droit et la Loi. Le juge et le jeu de la normalisation islamique du droit positif », Droit et culture, $\mathrm{n}^{\circ} 30$.

- 1996, « Représentation des répertoires normatifs en Égypte : les limites d'un consensus », Maghreb-Machrek, $n^{\circ} 151$.

- 1998, « Les formes du droit et les traces de l'histoire. La recherche constitutionnelle d'une moralité conforme ", Dakhlia J., Urbanité arabe, Paris, Sindbad.

Durkheim E., 1985 (1912), Les Formes élémentaires de la vie religieuse, Paris, PUF

(coll. « Quadrige »).

Eco U., 1990, The Limits of Interpretation, Bloomington, Indiana University Press.

Elster J., 1986, Le Laboureur et ses enfants. Deux essais sur les limites de la rationalité,

Paris, Minuit.

Esposito J., 1984, Islam and Politics, New-York, Syracuse University Press.

Ferrié J.-N. :

- 1995, « Réformisme, renaissance et contingence: la justification de la justice chez Nubar Pacha et Khayr ed-Din », A. Roussillon, Réforme sociale et mouvement national en Égypte, Le Caire, CEDEJ.

- 1998, « La Petite robe ou le dépassement des limites dans un régime de civilité », Hermès, nº 22.

Ferrié J.-N., Boëtsch G. \& Ouafik A., 1994, « Vécu juridique, norme et sens de la justice : à propos de l'avortement au Maroc ", Droit et Société, $\mathrm{n}^{\circ} 28$.

Ferry J.-M., 1992, « Qu'est-ce qu'une communauté politique ? », Cotereau A. \& Ladrière P., Pouvoir et Légitimité. Figures de l'espace public, Paris, Éditions de l'EHESS.

Geertz C., 1992, Observer l'islam, Paris, La Découverte.

Hourani A., 1983, Arabic Thought in the Liberal Age, Cambridge, Cambridge University Press.

Jacquemond R. :

- 1986, « Un projet de code pénal islamique égyptien », Bulletin du Cedej, n 20 ;

- 1994, «Quelques débats récents autour de la censure », Égypte/Monde arabe, $\mathrm{n}^{\circ} 20$.

Teubner G., 1993, Le Droit, un système autopoiétique, Paris, PUF.

Vatikiotis P. J., 1987, Islam and the State, Londres, Croom Helm.

\section{NOTES}

1.Comme le montre, par exemple, le débat américain sur la pornographie, e.g. Susan Eaton, The Problem of Pornography. Regulation and the Right ot Free Speech, Londres, Routledge, 1994.

2.Gâd al-Haqq 'Alî Gâd al-Haqq, décédé en 1995.

3.Entretien publié dans al-Ahrâm al-duwalî, 17/01/1992, traduit et cité par Bernard Botiveau (1993, p. 150). 
4.Ibid., p. 138.

5.Ibid., p. 140.

6.Ce que Bernard Botiveau définit comme « l'amalgame » du citoyen et du croyant (ibid., p. 138).

7.Arrêt du 18 mai 1996, traduit et analysé dans Dupret (1998).

8.Par réformiste, dans le contexte égyptien, j'entends : « modernisatrice » dans la tradition de Muhammad ${ }^{A} A b d u h$. On peut néanmoins douter que cette modernisation " scripturaire » instaura une véritable « modernité ». Sur ce point, les arguments de Geertz (1992) l'emportent sur les sornettes islamologiques qui soutiennent qu'une telle opinion « ne pourrait, éventuellement, être admise qu'au terme d'une démonstration [...] étoffée dont la matière devrait obligatoirement être fournie par une lecture approfondie des nombreux textes concernant sharî‘ a, usûl al-fiqh et fiqh laissés par les réformistes " (Chaumont, 1996, p. 66). C'est précisément la nécessité de justifier le droit à faire du neuf par référence à la Loi divine et aux fondements du droit - en d'autres termes, la justification du neuf par la prééminence du passé - qui est à l'origine de l'aporie réformiste. On ne peut donc situer le réformisme dans la postérité des Lumières comme s'emploie, par exemple, à le faire Hourani (1983). Les Lumières se fondent, au contraire, sur le rapatriement de la référence au sein de l'interaction sociale et non sur sa transcendance (Cf. Ferrié, 1995).

9.Il s'agit d'un exemple typique de ce que Sacks nomme une «paire relationnelle standardisée ».

10.Cf. Higâzî A. A.-M., al-Ahrâm, 06/01/1993, traduit et cité dans Jacquemond (1994, p. 33).

11.Cf. Mahfûz N., al-Ahrâm, 01/09/1994, traduit et cité dans Jacquemond (1994, p. 33).

12.Cf. Farahat M. N., al-Musawwar, 08/04/1994, traduit et cité dans Jacquemond (1994, p. 33-34). (Il va de soi que la juxtaposition des citations est à mettre au crédit de l'auteur de l'article).

13.Je suis ici la terminologie de Sacks qui nommait collection un ensemble de catégories considérées comme apparentées. Ainsi la collection « sexe » comprend (dans certains contextes) les catégories « mâle » et « femelle ».

14.Précisons que Jean-Marc Ferry ne conclut pas, pour autant, à la nécessité d'une position transcendante (Ferry, 1992, p. 123).

15.Il s'agit du magazine The Face, août 1997.

16.Relatée dans Jacquemond (1994, p. 28) Cf. aussi Akhbâr al-adab, n 31, 13/02/1994 et al-Ahrâm, 23/03/1994.

17.Cf. Dupret, (1995a, 1995b, 1996, 1998).

18.Cf. $v^{\circ} »$ Hadd », Encyclopédie de l'Islam (2 édition).

19.Ibid.

20.Bercher (1926), cité dans Bousquet (1990, p. 80).

21.Un coupable qui cherche à fuir doit être considéré comme étant revenu sur ses aveux (Bousquet, 1990, p. 80).

22.Le fondement dans l'antériorité couple les deux possibilités du «trilemme de Münchausen » décrit par Hans Albert (1968, p. 13 sqq.), dont la position est discutée dans Apel (1981). La troisième possibilité, l'aporie du cercle logique (ou de la circularité de la fondation), consiste à recourir, dans le processus de fondation lui-même, à des arguments fondés sur celui-ci. Toufefois, cette aporie ne représente pas un empêchement pragmatique. On peut, par exemple, redécrire le problème du cercle logique, en partant de la théorie de l'auto-référentialité des systèmes normatifs comme 
le fait Teubner (1993). Il relate l'histoire suivante : « Un rabbin connu pour la validité de ses opinions juridiques n'arrivait pas à rallier à son point de vue les autres rabbins assemblés à la synagogue. Il finit par invoquer le ciel et Dieu intervint pour confirmer son opinion, mais les rabbins ne s'y rallièrent toujours pas, en disant : "Nous ne tenons nul compte d'une voix divine, car Toimême, tu as écrit dans la Thora, au mont Sinaï, qu'il faut se rendre à l'avis de la majorité." Et Dieu rit, et dit : "Mes fils m'ont battu, mes fils m'ont battu." " (Teubner, 1993, p 7-8). Dans la perspective de Teubner, cette histoire illustre l'auto-référentialité de la norme, le niveau le plus haut de la hiérarchie des normes (Dieu) s'alimentant au niveau le plus bas, dans un processus de fondation circulaire. Mais elle peut aussi servir à rendre compte du primat de la contingence. En ce sens, un système de normes et sa clôture transcendante apparaissent d'abord comme le produit d'une forme de consensus. Que le primat de cet accord soit institué par la Loi divine est un subterfuge (nécessaire) comme l'appel à Dieu du rabbin, puisque des deux interventions divines - celle d'avant ou celle d'après - l'assemblée peut sélectionner la seule pertinente de son point de vue (une discussion de cette question figure dans Ferrié, Boëtsch \& Ouafik, 1994). Il est possible de déduire de cette histoire un certain nombre de choses dont la plus importante est assurément la différence séparant l'origine et la référence, en d'autres termes, l'indexation d'une chose ne livre pas sa provenance. Ce n'est pas la référence coranique qui fonde le droit d'une disposition ou d'un argument à prévaloir mais sa " communalité », c'est-à-dire la normalité du commun sur lequel elle se fonde.

23.C'est ainsi, par exemple, que John Esposito (1984), afin d'expliquer les relations contemporaines entre islam et politique, éprouve le besoin de retracer, dans deux longs chapitres, l'histoire de l'islam depuis la naissance du Prophète. On constate la même attitude chez Vatikiotis (1987, p. 19) qui fait précéder son analyse des problèmes nés de l'adoption de l'idéologie nationaliste européenne par les sociétés musulmanes, d'un chapitre sur la naissance de l'islam s'ouvrant par cette déclaration : « Il est utile de recapituler brièvement [...] l'histoire de la civilisation et des pays auxquels nous avons affaire quand nous considérons l'Islam et l'Etat-Nation. »

INDEX

Mots-clés : normalité, norme, censure 